\section{El "eterno retorno" de los esencialismos psicológicos: "indicios" del carácter}

The "eternal return" of psychological essentialism: "Clues" to character

María Christiansen*

Graciela Velázquez-Delgado**

\section{Resumen}

La historia de la psicología está saturada de concepciones esencialistas sobre la conducta humana, edificadas a partir de nociones como las de temperamento, fisiognomía, carácter y personalidad (que representan constructos

Departamento de Filosofía, División de Ciencias Sociales y Humanidades, Universidad de Guanajuato, Guanajuato, México. Correo electrónico: mariachr@ugto.mx

** Departamento de Historia, División de Ciencias Sociales y Humanidades, Universidad de Guanajuato, Guanajuato, México. Correo electrónico: gracevd@gmail.com ontologizados y naturalizados). En ese variopinto mosaico, la craneología (una forma específica del aún elogiado "cerebralismo") fue uno de los proyectos explicativos y predictivos de mayor envergadura durante el siglo XIX. Partió de supuestos materialistas, deterministas e individualistas, y validó una densa visión lineal del comportamiento. Su marco conceptual hizo del "indicio" un eje central de investigación, detección, interpretación y anticipación de las reacciones humanas. No obstante ocupar un lugar marginal en la historia oficial de la psicología científica, la craneología satisfizo funciones epistémicas pero también necesidades culturales dentro del nicho de creencias, prácticas y valores en el cual floreció (lo cual problematiza el recorte empobrecedor que resulta de narrar el pasado disciplinar desde un presentismo que minimiza, simplifica o ignora lo "pseudocientífico").

Palabras clave: esencialismo, conducta, craneología, indicio, individualismo.

\section{Abstract}

The history of psychology is full of essentialist conceptions about human behavior, built from notions such as temperament, physiognomy, character and personality (representing ontologized and naturalized constructs). In this motley mosaic, craniology (a specific form of yet acclaimed "cerebralism") was one of the explanatory and predictive larger projects during the nineteenth century. It embraced materialistic, deterministic and individualistic assumptions and validated a dense linear view of behavior. Its conceptual framework was based on "clues" as central pivot for research, detection, interpretation and anticipation of 
human reactions. Even though it occupied a marginal place in the official history of scientific psychology, craniology satisfied epistemic functions but also cultural needs within the niche of beliefs, practices and values where it flourished. This position problematizes the impoverishing cut that results from narrating the disciplinary past from a perspective that minimizes, simplifies or ignores the "pseudoscience".

Key words: essentialism, behavior, craniology , clue, individualism.

\section{Introducción}

De manera general, el término "esencialismo" se refiere al hecho de adjudicarle a las entidades una esencia o una naturaleza subyacente que las hace ser lo que son (Medin 1989: 1476). Si dicha entidad perdiera esas propiedades fundamentales, dejaría de ser tal. Así, desde una perspectiva semejante, se asume que, tras lo accidental y contingente, reside lo sustancial y necesario. La postura esencialista adquiere alta relevancia para la comprensión de las clasificaciones, ya que, al momento de categorizar, las similitudes de superficie son tratadas como indicadores heurísticos que permiten conocer los atributos recónditos y determinantes (Estrada y Oyarzún 2007). Por ello tal posicionamiento está estrechamente vinculado con el denominado "realismo taxonómico" (visión que afirma que los objetos que hallamos en el mundo están ordenados en grupos "naturales" o clases que existen antes de (a priori) cualquier intento de clasificación, y que dichas taxonomías pueden ser correctas (si se corresponden con las clases naturales) o incorrectas, en el caso de que tal correspondencia fracase).

A lo largo del tiempo, múltiples concepciones esencialistas han permeado las formas de pensamiento acerca de la conducta humana, dando lugar a la aparición, desaparición y reaparición de ideas, teorías, valores y prácticas diversificadas. El común denominador que dichos esencialismos comparten radica en conferirle importancia a rasgos que, a pesar de mostrarse como insustanciales, reúnen la mayor relevancia como indicadores de una realidad subyacente invariable, la cual permanecería oculta para los métodos de indagación inmediata. En tal marco, han concentrado un gran interés las amalgamadas nociones de "huella, rastro, síntoma, trazo, vestigio y señal", sobre todo porque el denominado "paradigma indiciario" las ha organizado conceptual y explicativamente. De acuerdo a los planteamientos de su máximo representante, el afamado historiador italiano C. Ginzburg, el tipo de saber obtenido a partir de "indicios mínimos" puede conducir al desvelamiento de fenómenos humanos más generales e incluso más ricos que cualquier codificación escrita (1999: 163). En realidad, este saber indicial, que las ciencias humanas comenzaron a afirmar recién en la década de 1870-1880, tenía, según Ginzburg, raíces muy antiguas:

Durante milenios el hombre fue cazador. La acumulación de innumerables actos de persecución de la presa le permitió aprender a reconstruir las formas y los movimientos de piezas de caza no visibles, por medio de huellas en el barro, ramas quebradas, estiércol, mechones de pelo, plumas, concentraciones de olores. Aprendió a olfatear, registrar, interpretar y clasificar rastros infinitesimales como, por ejemplo, los hilillos de baba (1999: 144). 
Siendo conjetural y basándose en datos secundarios o "bajos", el indicio pasó a verse como la puerta de entrada a misteriosas realizaciones humanas, aún cuando no se transmitiera por medio de libros, sino de viva voz, mediante gestos o miradas. Como bien aclara Ginzburg, los indicios "se fundaban en sutilezas que por cierto no eran susceptibles de formalización, que muy a menudo, ni siquiera eran traducibles verbalmente" (1999: 155).

En su exhaustivo análisis del paradigma indiciario, Aguirre propone la definición de "indicio" como:

[un] signo, o elemento, que siendo el resultado involuntario, o del despliegue y existencia de un cierto proceso o de una cierta realidad, o a veces de una creación inconsciente de su propio autor, se constituye en un dato que solo aparentemente es marginal o intrascendente, pero que analizado con más cuidado, se muestra como dato revelador de una realidad oculta, más profunda y esencial, realidad que no siendo accesible de un modo directo y evidente, y que poseyendo un comportamiento histórico que es incierto, no previsible y no deducible a partir de su propio pasado, sólo se revela mediante esos datos singulares y privilegiados, mediante esos "indicios", a aquellas miradas especialmente entrenadas y educadas para descifrar y escudriñar estos mismos datos reveladores (2007: 33).

Desde ese modelo de investigación, se supone que el conocimiento basado en la detección y desciframiento de "indicios" requiere de una experiencia práctica recurrente, puesto que la habilidad del observador adviene por una agudizada domesticación de la percepción, la intuición, la asociación, y otras destrezas similares (y no únicamente por la mediación primordial de teorías, abstracciones o generalizaciones). Por esta razón, el abordaje indiciario de la realidad social resulta practicado por un amplio rango de personas con trayectorias vocacionales distintas, desde el cazador, el carpintero, el marinero y el alfarero, hasta el médico, el juez, el detective, el político, el psicoanalista y el historiador. Por el hecho de tener una fuente eminentemente práctica, el saber indiciario anida más en la cultura popular y en las clases subalternas que en la cultura hegemónica. Como advierte Aguirre:

\begin{abstract}
¿Quién conoce mejor a la naturaleza, en sus dimensiones concretas y singulares, que el campesino que convive con ella a diario?, ¿y quién sabe más sobre los modos y comportamientos singulares del material y de los objetos fabricados, que sus propios creadores, los obreros de las fábricas?. ¿Y quién conoce mejor la ciudad que sus clases populares, que la viven, la sufren, la gozan, y la crean y recrean cada día sin cesar? Lo que no impide, naturalmente, que ese conocimiento de la naturaleza, de los productos o de la ciudad pueda ser parcial, incompleto y hasta en parte erróneo, pero igualmente legítimo y fundamental para, como dice Norbert Elias, "orientarse de manera funcional y práctica dentro del mundo", por parte de esas mismas clases subalternas (2007: 35).
\end{abstract}

Este último punto, que enfatiza la condición no deductiva del saber indicial (siempre falible y corregible), ha sido especialmente destacado por Ginzburg, al admitir que, más allá de que una pieza de evidencia histórica sea involuntaria (un cráneo, una huella, un vestigio de comida) o voluntaria (una crónica, un acta notarial, un tenedor), de cualquier manera requiere de "un marco interpretativo específico que debe estar relacionado con un código específico de acuerdo al cual la evidencia ha sido construida" (1991: 84). Atinadamente compara la evidencia de ambos casos con un cristal distorsionado: sin un análisis profundo de sus distorsiones inherentes (los códigos según los cuales se han construido y deben ser percibidos) una reconstrucción histórica del sonido es imposible (Ginzburg 1991: 84). 
A la luz de cambios interpretativos, "códigos que parecían impenetrables pueden ser eventualmente descifrados" (Davidson 2001: 152), emergiendo posibilidades hermenéuticas en principio insospechadas.

Otro aspecto que debe ser similarmente sopesado tiene que ver con que, a diferencia de la investigación del mundo natural, las realidades subterráneas que se desencubren mediante la guía del indicio albergan una ineludible carga de incertidumbre. Aguirre (2007: 36) advierte que el indicio abre la probabilidad de dar respuestas acertadas respecto a situaciones enigmáticas, como cuando el médico se pregunta cómo reaccionará el cuerpo ante un nuevo tratamiento, o cuando el líder popular se pregunta cómo reaccionarán las masas frente a una nueva medida represiva del poder, o cuando el pescador se pregunta cómo estará la pesca ese día, o cuando el carpintero y el alfarero se preguntan cómo responderán la madera y el barro a su trabajo, o cuando el juez o el historiador se preguntan cómo juzgar el nuevo caso o la nueva experiencia histórica bajo examen.

Ahora bien, a este interesante desplegado de ejemplos se agrega, sin duda, una pregunta que acecha no sólo al experto sino a la humanidad entera, a saber: ¿cómo reaccionará un individuo ante una determinada circunstancia?

El presente artículo se propone hacer una revisión crítico-reflexiva acerca de cómo, en el pensamiento científico social, han prevalecido ciertos supuestos epistémicos al intentar responder dicha pregunta. El objetivo principal es dar cuenta, desde el ejercicio filosófico e historiográfico, del poder de ciertas premisas para enquistarse en diferentes teorías acerca de la explicación/predicción de la conducta humana, imponiendo parámetros de comprensión asequibles únicamente desde una epistemología lineal, individualista y esencializadora.

\section{La conducta humana: Cuestión individual, mapeable y clasificatoria}

La intriga acerca de la conducta humana rebasa por mucho las fronteras del saber académico, y genera hipótesis explicativas que van desde las creencias más teorizadas y abstractas hasta las más rudimentarias e inocentes. No obstante, en ambos planos predominan relatos que invocan entidades cuya existencia permitiría entender por qué uno mismo o los demás actúan como lo hacen. Dos ejemplos clásicos lo representan: los conceptos de carácter y de personalidad, a partir de lo cual adquieren significatividad aquellos rasgos o "indicios" que conducen a determinar qué clase caracterológica o tipo de personalidad se halla en cada individuo. Las dos nociones son individualizantes y lineales en el sentido de que consideran que la base explicativa de la conducta humana reside efectivamente en el individuo, y que es éste quien modela las relaciones e interacciones (y no a la inversa). En gran medida esta cosmovisión se ha consolidado a raíz del peso aplastante que en nuestra cultura ha tenido (y tiene) la concepción sustancialista del ser humano como un "homo clausus" (Elias 1987: 34). Como parte del legado esencializador occidental, la filosofía moderna edificó la noción de "persona" en términos de un sí-mismo, un individuo cerrado dentro de su invisible y almidonada muralla identitaria. Dicho de otra manera, la capacidad para el pensamiento relacional, conector y recursivo se ha ido atrofiando bajo el poderosísimo influjo de 
la imperante epistemología lineal e individualista, arraigada tanto en las hegemónicas posturas filosóficas (el ego cartesiano, la mónada leibniziana, el ego solipsista kantiano), como en las definiciones cristianas de "persona" como "sustancia racional, indivisible e individual" (Cassiodorus Cit. en Jimeno 2002: 3). En consecuencia, el hecho de considerar que la conducta de un individuo queda definida por su "carácter" o por su "personalidad" suena "natural" y consistente con la creencia de sentido común de que "cada persona es un mundo".

A la luz de la combinación de un vasto conjunto de premisas escasamente reflexionadas, fue posible que germinara la creencia de que el "examen indiciario" podía fungir como un recurso útil para adivinar la constitución psíquica de un individuo. En dicho marco, la topografía corporal se constituyó como un referente idóneo. Abundan, en el registro histórico de las ideas, los escritos de quienes pretendían demostrar que el carácter, las posibles reacciones conductuales e incluso el destino de un individuo podían inferirse a partir de un conjunto de signos o huellas a descubrir e interpretar. De manera que la noción de una "cartografía del cuerpo" gozaba de un importante atractivo que alcanzó un momento de esplendor en el último cuarto del siglo XIX. Como bien señala Perazzi (2009), esa fue la época de las grandes investigaciones de la morfología humana y de la consagración de las técnicas de observación y desciframiento. Lo que guiaba las multiplicadas indagaciones en tal dirección era el principio según el cual "cada individuo, aunque sujeto a normas de clasificación estandarizadas, era portador de "signos característicos" (señas particulares) que lo tornaban una entidad psicofísica única e indivisa" (2009: 122). Que los "signos exteriores" expresaban el estado psíquico del individuo respondía a un "consenso no argumentado", como tantas creencias que hacen época y que raramente son revisadas con filo crítico y cuestionadas.

El deseo y la necesidad de someter a contrastación la pericia del experto en la detección de "indicios" fomentó el interés en los espacios que reclutaban, por diferentes motivos, personas de muy diversa índole. En ese sentido, las grandes ciudades facilitaban mezclas de caracteres que las convertía en un laboratorio ideal (ya que el sujeto observado se hallaba en una situación natural, no controlada y por lo tanto no contaminada por la presencia del observador). Cuanto más demográficamente mezclado fuese un lugar, más poder de seducción ejercía para el observador entrenado. Por ejemplo, una de las urbes más reconocidas en el mundo occidental del siglo XIX era Londres, una ciudad admirada por su gigantismo, su heterogeneidad poblacional, su cosmopolitismo y sus contrastantes estilos de vida. Los analistas sociales se referían a ella en términos de una "síntesis del mundo", un observatorio desde el cual se podía percibir "una enorme variedad física, mental y ocupacional sin igual en el resto del planeta" (Patterson 1854). El adulado cronista de The Morning Chronicle, H. Mayhew, describía a Londres como un "vivarium", un universo racial coexistiendo dentro de los límites metropolitanos, y comparaba un paseo por sus calles con "una excursión geográfica a través de las múltiples regiones del globo" (Mayhew y Binny 1862: 59) ${ }^{1}$. Una desconcertante variedad de caracteres humanos ahora se

Sobre Londres y otras ciudades victorianas, se recomienda consultar Kingsley (1859), Brabazon (1881), Beaver (1970), James (1978), Watkin (1982), Olsen (1986), Elliot (1986), Fuller (1989), Carré (1989), King (1995). 
reunía en lugares comunes y las diferencias individuales comenzaban a ser objeto de curiosidad antropológica, de ordenamiento, de codificación y de clasificación de los habitantes (una versión "localizada" de lo que el etnólogo y el antropólogo hacían a gran escala). En enero de 1866, el Popular Magazine of Anthropology hacía su primera aparición advirtiendo al lector sobre el hecho de que:

Nuestras propias islas, nuestras grandes ciudades y distritos, le dan al antropólogo un importante campo de estudio (...) La naturaleza compuesta de nuestras poblaciones (...) constituye un tópico de importancia capital. Hay, entre nosotros, tipos humanos que contrastan tanto como los que podrían ser observados en cualquier rincón de la Tierra. El escandinavo, el sajón, el celta, el romano, el bretón, a quienes nos esforzamos por clasificar y ordenar, viven entre nosotros y expresan mejor que nunca sus cualidades morales y mentales (...) Hay cualidades naturales tan inherentes al hombre de las islas británicas como aquellas del habitante de Caffre, Málaga o la Patagonia².

Nótese aquí que la industrialización y la concomitante urbanización habían traído consigo la constante exposición de la diversidad humana, abriendo un gran abanico de interrogantes acerca del carácter, la conducta y el lugar "natural" de cada sujeto que formaba parte de las multitudes. El hecho de mezclar individuos de clases sociales diferentes en sitios estratégicos (la estación del ferrocarril, las áreas de esparcimiento, la taberna, el mercado, las calles comerciales, los espacios deportivos, entre otros) facilitaba las contrastaciones, y permitía cotejar cuánto diferían en sus rasgos físicos, en sus formas de hablar y de gesticular, en sus posturas, sus formas de vestir, sus hábitos de consumo, sus preferencias, sus

(1866) "Anthropology: A Practical Science", Popular Magazine of Anthropology I: 7-8. gustos, sus creencias, sus temores, sus expectativas, etcétera. ¿Por qué los seres humanos eran tan distintos entre sí? ¿Ocupaba cada quién un rol predeterminado? ¿Era la educación recibida lo que los diferenciaba? ¿Se modificaban sus conductas tras vivir largo tiempo en la ciudad? ¿Existía una relación entre sus comportamientos y su apariencia física (color de piel, rasgos faciales y craneales)? ¿De qué dependía el futuro de cada uno de ellos?

Todas estas preguntas implicaban el estudio comparativo de conductas, actitudes y patrones de reacción observados pacientemente a lo largo del tiempo. Numerosos microespacios citadinos se convirtieron, así, en nidos de práctica indiciaria compartida por varias disciplinas, y estructurada en función de una doble comparación: de los seres humanos entre sí y de éstos con los animales. Usualmente, las marcadas diferencias entre los individuos eran explicadas en función de 1) desigualdades naturales, innatas; 2) desigualdades ambientales, suscitadas por exposición a un medio particular (polémica conocida en la historia de la ciencia por su expresión inglesa: "Nature Versus Nurture"). Además, se suponía que la doctrina que podía explicar las diferencias entre los caracteres individuales servía también para entender las diferencias entre los "caracteres nacionales".

Entre las posiciones que daban cuenta de las diferencias individuales en términos de una desigualdad innata, se hallaba la antigua doctrina de los temperamentos (basada en la concepción galénica de los humores), según la cual los rasgos psicológicos y físicos de un individuo se debían a un tipo temperamental definido, determinado por la predominancia de un fluido específico (sanguíneo, flemático, 
melancólico y colérico). A lo largo del siglo XIX esta teoría conservó, en algunos casos, su tipología original (por ejemplo, en el trabajo de J. Elliotson (1835)), mientras que otros la modificaron de acuerdo a nuevos conocimientos sobre el sistema nervioso (como fue el caso de A. Bain (1861) y H. Maudsley (1867) ${ }^{3}$.

La clásica concepción temperamental favoreció el desarrollo de una gran variedad de métodos "inductivos", a partir de los cuales se pretendía discernir y predecir el carácter y la trayectoria vital de una persona. La "fisiognomía" era, probablemente, la práctica más popular y se basaba en la idea de que los rasgos faciales, así como la forma de la cabeza y el cuerpo eran, en un sujeto, reveladores de su carácter. Tanto la fisiognomía como otras prácticas afines (la patonomía, la metospocopia, la quiromancia) pretendían ser la "llave maestra con la cual abrir el místico tesoro del alma" (Jackson 1863: 131), una "herramienta útil en la traducción de las disposiciones, capacidades y temperamento de un sujeto" (Carson 1868: 24). La patonomía consistía en la lectura de las emociones en las expresiones faciales; la quiromancia leía el carácter a través de las líneas de la mano y la metospocopia lo hacía por medio de las líneas del rostro.

Varias de las ideas temperamentales y fisiognómicas tradicionales fueron absorbidas, en la década de 1860, por las teorías raciales desarrolladas en el seno de la etnología y la naciente antropología 4 . Sus categorías

Ver una descripción de cada uno de los temperamentos en Elliotson (1835), Castiglioni (1947), Singer y Underwood (1962), Haley (1978).

De hecho, J. Hunter (presidente de la Sociedad de Antropología, fundada en 1863 al separarse de la Sociedad Etnológica de Londres, presentaba a la antropología como "la fusión entre la anatomía, la fisiología, la patología, la psicología y la fisiognomía" (1867: VI, 329). Ver también Stocker (1900), Stocking (1987), Curtis (1971), Gould (1982), Cowling (1989). de clasificación daban cuenta de que las características físicas, psicológicas y emocionales de los miembros de una cierta clase social estaban, "por supuesto", asociadas con su fisiognomía y que ésta podía explicarse en términos de su origen racial (que, para algunos como J. Murray (1870: 24), explicaba la constitución temperamental). Por ejemplo, se hablaba de las clases gobernantes como portadoras de rasgos heredados de los normandos invasores, de la clase media como representando un híbrido entre los teutones y los sajones, y de las clases bajas como descendientes de los celtas.

Estas prácticas de lectura del carácter no eran simplemente descriptivas. Por el contrario, imponían parámetros de desarrollo con los cuales determinar el grado de evolución de un individuo, raza o nación. Las formas y proporciones de la cabeza y la cara eran consideradas como las marcas más distintivas entre animales y humanos. Una fisiognomía "correcta" implicaba una cabeza en la cual el cráneo estaba bien formado, y la frente y el mentón trazaban una línea perpendicular ("ortognatismo"). Inversamente, los rasgos físicos de una fisiognomía "incorrecta" ("prognatismo") eran, principalmente, una mandíbula grande y proyectada, y una frente pequeña y retroyectada, lo cual (dada la semejanza con la fisiognomía animal) indicaba un bajo rango en la escala de perfección. Aquellos considerados como inferiores en la jerarquía social (criminales, mujeres, niños, páuperos, delincuentes, insanos, campesinos, e irlandeses, entre otros) eran metafóricamente igualados con las razas menos desarrolladas, "primitivas" y "salvajes". La superioridad evolutiva era ejemplificada con la fisiognomía de la raza caucásica, mientras que la inferioridad 
tenía como referente a la fisiognomía de la raza $\mathrm{Negra}^{5}$. En el marco de la teoría racial y del evolucionismo darwiniano, estos supuestos fisiognómicos recuperaron gran popularidad (como lo sugieren las múltiples ilustraciones de algunas revistas de gran circulación entre la clase media elevada ${ }^{6}$, así como las novelas victorianas y las obras artísticas). Formas, tamaños y proporciones de la nariz, de la boca, de las manos, de los pies, del tórax, y hasta de la forma de caminar, se suponían "reveladores de carácter".

No obstante la existencia multifacética de posiciones esencializadoras, existía una coincidencia muy extendida sobre la idea de que el órgano caracterológicamente más "informativo" era el cráneo (parte fundamental del esqueleto), conformado por la cara ("fisiognomía") y por el cerebro ("cápsula"). De ahí que la doctrina más representativa por mucho tiempo fuera la craneología (Perazzi 2009: 130). El cerebro era tan importante como el tamaño, forma y proporción de la cara y la cabeza. Este aspecto fue subrayado por la más emblemática de las teorías clasificatorias de los tipos humanos durante la primera mitad del siglo XIX: la frenología, una doctrina del carácter basada en la ya existente craneología, y que, al igual que ésta, tomaba la forma y tamaño del cráneo como indicio caracterológico (independientemente

Para cotejar comparaciones, se puede consultar Elliotson (1835: II), Prichard (1843: I), Morton (1854). Acerca de las analogías entre la población inglesa y las razas, ver Spencer (1855: II), Davis y Thurman (1856), Mayhew y Binny (1862), Mac Lennan (1865), Lubbock (1870). Sobre la etnología y la antropología de la época, ver, por ejemplo, Napier (1867, 1870), Irvine (1955), Lubbock (1865), Curtis (1971), Heuman (1981), Gould (1982), Mac Dougal (1982), Baucom (1999)

Las revistas londineneses más famosas eran Punch, Fun y London Illustrated News. Sobre 'fisiognomía de clases' ver Wells (1866) y Curtis (1971). de las conductas observables de un sujeto). Tras ser exportada desde Austria (donde la había fundado, en el siglo XVIII, el médico vienés Franz Gall (1825), quien originalmente la llamó "organología" o "fisiología cerebral"), la frenología entró en Inglaterra con gran ímpetu, siendo a veces nombrada como "psicología de las facultades" o "ciencia de la mente". A continuación, se expondrán sus principales premisas y su intersección con otras disciplinas indiciarias afines, de las cuales (y a las cuales) retroalimentó.

\section{Frenología: Una psicología de las diferencias individuales}

Los principios frenológicos básicos se pueden enumerar como siguen:

1. El cerebro es el órgano de la mente.

2. La mente está compuesta de facultades innatas, múltiples y distintas.

3. Dado que son distintas, cada facultad debe residir en una región cerebral separada.

4. El tamaño de un órgano es una medida de su poder.

5. La forma del cerebro está determinada por el desarrollo de varios órganos.

6. Puesto que el cráneo toma su forma del cerebro, la superficie craneal puede ser leída como un índice exacto de tendencias y aptitudes psicológicas ${ }^{7}$.

Una explicación detallada e ilustrada sobre estas tesis se puede encontrar en Waller (2001), asimismo en Van Wyhe $(2002,2003)$ y Hilts (1982) 
El conjunto de estos principios implicaba que, al examinar, por tacto frenológico, la forma e irregularidades de una cabeza o cráneo, uno podría descubrir el desarrollo de órganos cerebrales responsables de diferentes rasgos del carácter. Por ejemplo, una protuberancia en la frente en la posición atribuida al órgano de la benevolencia supuestamente revelaba que el individuo tenía bien desarrollado dicho órgano, y que, por tanto, su conducta sería manifiestamente benevolente.

Vista como una ciencia que postulaba la localización cerebral de los rasgos del carácter, la frenología fue ganando espacio en la sociedad inglesa cuando un discípulo de Gall, llamado J. Spurzheim, la introdujo en Edimburgo, alrededor de 1815. La recepción de su doctrina fue ambivalente, pero en términos generales sus escritos $(1821,1825)$ desataron un gran entusiasmo entre sectores de la clase media conformados por quienes no hallaban lugar en los espacios académicos dominados por el elitismo intelectual. Entre ellos se destacó G. Combe, quien publicó diversos escritos (1825, $1827,1836,1840)$, entre los cuales se hallaba uno de los libros mejor ponderado y vendido en la época: Constitution of man. En poco tiempo, los frenólogos difundieron su ciencia a lo largo de Inglaterra, fundaron sociedades frenológicas, lograron incluso la adhesión enérgica de algunos médicos muy prestigiosos, como fue el caso de J. Elliotson, y contaron con el apoyo de órganos editoriales que servían de vehículo para difundir sus teorías y consolidar sus prácticas (Phrenological Journal, MedichoQuirurgical Review, entre otros).

Desde el punto de vista ideológico, los frenólogos reunían las típicas características de los reformadores políticos y sociales, llegando a asumir, en algunos casos, posturas ultrarradicales. Esto le dio a la frenología inglesa un aspecto peculiar con respecto a sus equivalentes europeas, ya que mientras estas últimas eran marcadamente deterministas, la primera se presentaba como un conocimiento de la naturaleza humana que, además de exponer la verdadera doctrina acerca de la conducta, era el medio adecuado para modelar y transformar el comportamiento, y para mejorar las condiciones de la vida moral, laboral, afectiva, marital, profesional, política, etcétera. Se suponía que el tacto o masaje craneológico (especialmente en la infancia más temprana) podía llegar a moldear y alterar el desarrollo craneal y mitigar el intransigente materialismo determinista que, doctrinalmente, la caracterizaba. En la época en que tuvo su mayor auge (desde 1820 a 1840), el frenólogo era consultado por personas que pretendían estimular 0 inhibir ciertas capacidades en sus hijos, o por familias de parejas que iban a contraer nupcias y se interesaban en conocer el carácter de sus futuros cónyuges y descendientes, o por los empleadores que deseaban conocer el perfil de algún potencial empleado, o por políticos que usaban su mapa frenológico para hacer proselitismo, o por defensores de la reforma del sistema penal que se valían de la explicación frenológica de la conducta criminal, entre muchas otras ocurrentes aplicaciones.

Sin embargo, la frenología también atrajo a quienes compartían ideas más conservadoras. Numerosos sectores evangélicos recurrían al discurso de las facultades frenológicas para aumentar la evidencia a favor del creacionismo (por ejemplo, la existencia y el desarrollo de la facultad de veneración). Sin embargo, sería posible generalizar este fenómeno, ya 
que hay que decir que una gran cantidad de frenólogos eran promotores de doctrinas desacralizadoras que cimentaron las bases tanto para el advenimiento de la incipiente filosofía de la autoayuda como también del naturalismo científico, tendencias distintivas del pensamiento inglés secularizador.

Ahora bien, ¿qué tan popular era la frenología? ¿Cómo se situaba con respecto a los sistemas de pensamiento que los victorianos consideraban como válidos y razonables? ¿Consistía únicamente en un esbozo explicativo y predictivo de la conducta humana, o era, más bien, una cosmovisión a través de la cual se organizaba, se percibía y se ordenaba una inmensa variedad de tipos humanos y clases sociales?

Cuando la lupa historiográfica es colocada sobre un dominio más amplio que el comprendido por las obras magistrales de aquellos intelectuales científicos que varios historiadores ortodoxos han estimado como únicos merecedores de un lugar en la historia de la psiquiatría, y cuando dicha lupa se sitúa más allá de los márgenes de la ciencia "oficial" y enfoca dominios culturales menos expuestos, es posible ver que las creencias frenológicas impregnaban los procesos de codificación y clasificación de los seres humanos, dentro y fuera de la ciencia. Pero, para recuperar el sentido que la frenología tuvo en el mundo victoriano, es preciso volver al pasado evitando transitar por los caminos normativistas habituales que tienden a observar el corpus frenológico desde la estrechez de la dicotomía "ciencia - pseudociencia". Evaluar la validez de la frenología recurriendo a cánones epistémicos actuales ("presentismo") implica entender que la relevancia y la fundamentación de los conocimientos no son independientes de los "ecosistemas" que los mantienen vigentes. En tal sentido, las aludidas prácticas caracterológicas de corte indiciario que se han venido revisando no podrían ser justamente valoradas fuera de su particular y único andamiaje simbólico e interactivo.

\section{El conocimiento frenológico en su con- texto histórico indicial}

En primer lugar, es preciso tener presente que la frenología, lejos de ser un saber aislado, estaba inmersa en una nutridísima fuente de métodos caracterológicos de larga data (entre ellos los antes mencionados: la patonomía, la quiromancia, la metospocopia, como también otros menos divulgados pero igualmente importantes, tal como la osfresiología (o clasificación odorífica) ${ }^{8}$.

Dada la naturalización de muchas creencias atrincheradas en la tradición fisiognómica (sobre todo en torno a las nociones de ortognatismo y prognatismo, traducidos en términos de superioridad $o$ inferioridad en la escala de la naturaleza), las publicaciones de la época reflejaban (y a la vez reforzaban) la idea de la apariencia física como "mapa" del carácter y del comportamiento. Por ejemplo, en Types of humanity (1854), S. Morton comparaba las cabezas y cráneos de Apolo (representando al "mejor tipo" de la raza caucásica), con las cabezas y cráneos de un negro y un chimpancé. Asimismo, proliferaban las ilustraciones cotejando los perfiles de reconocidas figuras históricas con los de "razas inferiores" y animales. Luego de que Darwin popularizó (en

Para detalles sobre la osfresiología, consultar (Corbin 1987 Cit. en Perazzi 2009: 134). 
$1859,1871,1872)$ la idea de que los humanos descienden de los monos, el prognatismo devino -en tanto indicador de bajo desarrollo- una característica de primitivismo que las revistas ilustradas de mayor prestigio, como Punch y Fun, se encargaban de poner en circulación. A modo de ejemplo, cabe mencionar que en 1866 el famoso fisiognomista S. Wells comparaba la fisiognomía de importantes teólogos británicos (Emmanuel Swedenborg, John Wesley, Thomas Chalmers, entre muchos otros) con la de destacados boxeadores como Bob Travers, Tom Sayers y Tom King. En dicha ilustración los clérigos aparecían con una frente expandida y un ápice craneal elevado, rasgos propios de un alto desarrollo moral. La cabeza del boxeador, en cambio, era más amplia en la base (región de las tendencias animales, tales como la combatividad y la destructibilidad), con un deficiente desarrollo de las cualidades morales e intelectuales (bajo ápice craneal, frente retroyectada). En el caso de grandes líderes de la vida nacional (especialmente los de prestigiosa carrera militar) se señalaban otros atributos, además de las constantes expectativas de cabezas grandes y bien proporcionadas (mostrando buen desarrollo cerebral); por ejemplo, un amplio desarrollo pectoral era revelador de fuerza tanto corporal como mental, así como de inigualable energía y determinación.

Es pertinente insistir en que la clave de la perfección fisiognómica residía en el distanciamiento que ciertos rasgos imponían entre el reino humano y el reino animal. Aunque los aspectos faciales y craneales eran los más reconocidos, otros rasgos físicos eran igualmente tenidos en alta estima como "indicios" caracterológicos. Tal era el caso de la clase de perfil de un individuo, a partir de lo cual podía descifrarse su fuerza de carácter (Warwick 1864). La nariz, cuyo desarrollo ideal era expuesto por los perfiles aquilinos, agregaba intensidad de carácter. Un puente nasal prominente, nariz "romana", era un rasgo inherente a caracteres dominantes (casos señalados como "típicos" eran los de Julio César y el Duque de Wellington), aptos para liderar un ejército, o para dirigir los asuntos públicos. En cambio, la refinada y delicada nariz "griega" (aquella que continuaba en una línea ininterrumpida desde la frente) indicaba un temperamento poético o artístico. La nariz "racionalista", amplia en su base, reflejaba importantes poderes de meditación. Pero, curiosamente, la nariz chata y respingona no era clasificada ya que no indicaba presencia sino ausencia de capacidad para el raciocinio. Una nariz poco prominente contribuía a un perfil desdibujado, que "evidenciaba" un escaso desarrollo evolutivo asociado a individuos "poco dignos" y ubicados en los estratos sociales inferiores (tal clase de nariz era más tolerable en las mujeres, consideradas como seres subdesarrollados). Los despreciados irlandeses eran vistos como portadores prototípicos de esta clase de nariz, y la prensa solía mofarse de las derivaciones comportamentales que esto supuestamente acarreaba.

Otro rasgo facial de gran importancia caracterológica era la boca, capaz de representar un carácter "refinado" o, por el contrario, "apetitoso". Como sostenía un distinguido médico victoriano, en los dos labios se repetía la oposición de la mitad superior y la mitad inferior de la cara. En el labio superior se resumían las propiedades del carácter psíquico superior; en el labio inferior, aquellas de la naturaleza corporal; así, era esencial para una cara "noble" que el labio superior gobernara al inferior (es decir, que no lo sobrepasara en tamaño). Desde el punto de vista fisiognómico, 
los labios gruesos eran propios de una persona entregada a la sensualidad y a la indolencia, manifestadas en el hábito de consumir demasiado tabaco, beber desmesuradamente, y vivir una vida viciosa.

Ahora bien, la práctica de las clasificaciones fisiognómicas, apoyada en las analogías tanto como en la anatomía comparativa, no se reducía al examen de la cabeza y de los rasgos faciales, sino que se extendía al cuerpo, tanto en sus formas como en su expresión. Desde el estilo con el que alguien caminaba hasta la forma, tamaño y proporciones de la mano, eran objeto de discusión científica del carácter. Por ejemplo, en la década de 1860, circulaban varios escritos que renovaban las apelmazadas teorías quirománticas. En 1865, se había reeditado un escrito de M. D'Arpentigny y Desbarrolles, traducido al inglés por R. Beamish en 1843, con el nombre de Psychonomy of the hand, or the hand: An index to mental development. La distinción principal entre una mano ideal y una mano fisiognómicamente indeseable se trazaba, nuevamente, sobre una base anatómica. Cuanto más dominante (en tamaño) fuese la palma de la mano con respecto a los dedos, mayor sería la aproximación a la brutalidad, a los bajos instintos y a la degradación moral. Se asumía que los dedos eran la herramienta de la vida intelectual, mientras que la palma lo era de la vida animal. El pie recibía un tratamiento similar. La pequeñez y la delicadeza de la mano y el pie se estimaban como signos de un desarrollo y un refinamiento superior, a la vez que sugerían una posición avanzada en la cadena evolutiva (Craig 1867, 1884).

¿Qué alcance tenían las clasificaciones de los seres humanos de acuerdo con estos sistemas de conocimiento que, como ya se dijo, serían subsumidos por la teoría racial incubada en la embrionaria antropología victoriana? Para responder a este interrogante es preciso insistir en que la fisiognomía (en todas sus variantes, incluida la frenología) no era únicamente un molde perceptual que configuraba las observaciones e interpretaciones que los etnólogos hacían en otras culturas diferentes a la europea, sino que era, ante todo, una constelación de esquemas conceptuales desde los que se definía, se explicaba y se predecía el carácter, la conducta y el destino incluso de la misma sociedad inglesa. Dicho de otra forma, la fisiognomía y la frenología lograban explicar las diferencias de carácter a través de una infraestructura teórica y práctica que ontologizaba a dicha entidad (esto es, le confería al carácter un estatus de "realidad"). Los vívidos discursos sobre su conformación y la progresista retórica clasificatoria a la que daba lugar facilitaba asimismo su "naturalización" (es decir, devino posible pensar y hablar del carácter como algo originalmente "dado"). Esta cosmovisión permitía, además, proponer un marco de inteligibilidad para sustentar la creencia de que cada individuo, cada familia, cada estrato social, cada nación, cada raza, llevaban "inscrito" su destino. Sólo era cuestión de explicitar aquello que los indicios tímidamente anunciaban.

La razón de la fecundidad que estos conocimientos adquirieron en Inglaterra responde más a las necesidades culturales del momento que a razones epistémicas. $\mathrm{O}$, en todo caso, valdría decir que hay entre sendos aspectos una interesante relación de reciprocidad y retroalimentación. Cabe preguntarse si las prejuiciosas ideas sobre la conducta humana que fermentaron en la Inglaterra decimonónica hubieran visto la luz en 
un mundo rural ${ }^{9}$. Está claro que, dentro de los límites metropolitanos, era posible encontrar la heterogeneidad humana que los etnólogos, antropólogos y viajeros hallaban alrededor del mundo. También está claro que, ante la inexorable demanda de ordenamiento impuesta por la vida moderna, lo real y lo imaginario se volvían prácticamente indiscernibles, y que los métodos de clasificación y distinción de individuos, grupos y clases adquirían un sentido prioritariamente instrumental. Toda acción, actividad o evento que reuniera aglomeraciones (y que, en consecuencia, propiciara la mezcla de caracteres de diferentes grupos humanos) generaba, en el análisis cultural, una similar necesidad de determinar si tal aglutinación implicaba riesgos de homogeneización o nivelación de caracteres. El fenómeno de las "muchedumbres" (la fábrica, el transporte, el mercado, las escuelas, las manifestaciones, los parques públicos y muchos otros eventos impensables en el viejo estilo de vida agraria - pueblerina) provocaba gran confusión acerca de la cuestión de cómo afectaría tal exposición al carácter de cada individuo. En On liberty (1859b), John Stuart Mill refleja el tipo de preocupación que varios intelectuales se hacían ante el inexorable advenimiento de esta sociedad de masas. Mill advertía de los potenciales efectos de mediocrización y "achatamiento" del carácter del individuo perdido entre un gran número y esclavizado por la opinión pública. Al respecto, decía Mill (1859b: 137): "La creciente tendencia a la uniformidad

Acerca de las características de las ciudades inglesas, en especial de Londres, y sobre el estilo de vida impuesto por la industrialización, es orientador consultar la siguiente bibliografía: Mayhew (1849, 1874), Youmans (1867), Richardson (1876), Rasmussen (1934), Houghton (1957), Woodward (1962), Reader (1964), Briggs (1970), Best (1971), Seeley (1971), Stedman Jones (1971), Dyos y Wolf (1973), Dyos (1982), Dennis (1984), Olsen (1986), Thompson (1988), Carré (1989), Baucom (1999). en Europa, bajo el "régimen moderno de la opinión pública", hace que Europa marche decididamente hacia el ideal Chino de hacer a todo el mundo parecido"10. Tratándose del carácter, lo que se estaba poniendo en boga era, según Mill, "no tener carácter alguno".

Ahora bien, no hay que perder de vista que la opinión de Mill, como fiel adherente al asociacionismo anti-frenológico, no representaba la de la mayoría de sus contemporáneos notablemente convencidos de que la mezcla eventual de clases sociales y caracteres no suponía una confusión del lugar que cada quien ocupaba en la jerarquía social. Si el carácter estaba impreso en la fisiognomía de cada individuo, ninguna mezcolanza podría alterar notoriamente el trabajo de la naturaleza. En su influyente London Characters (1849), Mayhew dejaba constancia de su reconocimiento simple y espontáneo de las diferentes clases de caracteres que poblaban los espacios urbanos. Cualquier persona entrenada para la destreza fisiognómica podría identificar rápidamente a los individuos "indeseables" y "peligrosos", independientemente de la densidad numérica de una multitud

De estos supuestos esencialistas no quedaron inmunes ni siquiera la literatura y el arte de la cultura victoriana. La novela (Ch. Dickens, E. Gaskell, J. Kanvanagh, W. Collins, G. Eliot

- Es preciso aclarar que, si bien Mill lamentaba el poder nivelador de la sociedad de masas y su concomitante degradación de las diferencias individuales, sin embargo, no abogaba por un enfoque frenológico de la naturaleza humana (Mill 1859a). Por el contrario, tanto él como su discípulo Alexander Bain seguían adheridos enérgicamente al ambientalismo tradicionalmente defendido por el asociacionismo inglés. Para tener una idea cabal de la postura que los asociacionistas asumieron frente a las tendencias frenológicas es necesario consultar la -poco conocida- obra de Bain de 1861. Un enfoque integral sobre el tema se encuentra en Christiansen (2009) 
y Ch. Kingsley, entre otros), la caricatura (J. Leech), las ilustraciones en las revistas (Punch, Fun, London Illustrated News), las pinturas (especialmente W. Frith) y prácticamente toda manifestación artística de la naturaleza humana expresaba un cierto compromiso con alguna de tales premisas ${ }^{11}$. En las representaciones visuales, ciertas asociaciones perceptuales eran constantes, como por ejemplo las clases bajas irlandesas evidenciando su anticipada fisiognomía prognatista, indicadora de su "desafortunado desarrollo" (lo cual se expresaba frecuentemente en términos del grotesco "tipo bull dog", clásica en la fisiognomía criminal). Aludiendo a su escasa evolución, acotado refinamiento y atrofiada sensibilidad, St. James Chronicle se refería a ellos como "demacrados", con un rostro "tosco e inanimado, indicativo de una naturaleza bruta". El St. James se animaba incluso a predecir el background social de esos sujetos, así como sus malos hábitos y sus probables carreras en la criminalidad y la vagancia, concluyendo que había "pocas esperanzas para su recuperación, dado su aspecto y degradación" (17 de abril 1862: 8). El Art Journal los identificaba como "individuos cuyo semblante los ha marcado como eternos reprobados" (3 de mayo 1862: 123).

El medio siglo victoriano estuvo, así, repleto de teorías clasificatorias acerca de la naturaleza humana, anticuadas o nuevas, desgastadas o renovadas, pero todas ellas aunadas bajo una compartida pretensión de cientificidad. Ciertamente la concepción racial fue la que

\footnotetext{
Sobre el acato a las reglas fisonómicas en las novelas, ver Tytler (1982), donde analiza la obra de los escritores mencionados. Un análisis de la incorporación de las reglas fisonómicas en las pinturas victorianas se halla en Cowling (1989). Ver comparaciones ilustradas sobre fisiognomía humana y animal en Paget (1856), Warwick (1864), Beamish (1865), Wells (1866).
}

adquirió protagonismo, pero no sin la influencia de ciertas ideas, métodos y técnicas frenológicas y fisiognómicas. Aquellos estereotipos de los irlandeses, de los obreros, de los criminales, de los insanos y de los campesinos, resaltando el hecho de su involucionada fisiognomía prognatista, recibía, en el contexto de la descollante antropología, una interpretación racial que la asociaba con la naturaleza aborigen y la complexión oscura (cabello-ojos) usualmente atribuida a los celtas. J. Beddoe, por ejemplo, había inventado una escala para medir el grado de negritud de las razas de Gran Bretaña, y correlacionaba el mayor nivel de oscuridad con un tipo "africanoide"/prognatista de celta. Por otra parte, las explicaciones raciales incorporaban no sólo elementos de la antigua teoría temperamental hipocrático-galénica, sino también de la teoría del ángulo facial de Peter Camper (la cual había sido esbozada desde fines del siglo XVIII, y que explicada las diferencias raciales a partir de los ángulos faciales obtenidos de trazar dos líneas: una desde el orificio de la oreja hasta la mandíbula superior, y la otra desde la frente hasta la mandíbula superior). Este médico argüía supuesta evidencia anatómica para "comprobar" que el ángulo facial de los negros se alejaba del característico en el hombre blanco. Según Camper, los europeos presentaban un ángulo de $80^{\circ}$ (sumamente deseable), mientras que los africanos presentaban un ángulo de $70^{\circ}$ y el orangután de $58^{\circ}$. Un adecuado ángulo facial (como el europeo) era "indicador" de cualidades morales e intelectuales socialmente respetables (Urteaga 1993).

Sin duda alguna la teoría evolucionista contribuyó significativamente a sancionar biológicamente estas preconcepciones, ya que en dicha teoría la subestimada fisiognomía prognatista tenía una importancia central. Lo mismo se constata con respecto al 
evolucionismo social de pensadores como Mac Lennan (1865), en quienes el concepto de "primitivismo" remitía al prognatismo y a otras ideas de origen fisiognómico-frenológico. Más aún, un estudio minucioso de los trabajos del primo de Darwin, F. Galton (1865, 1869), deja ver cómo su defendido hereditarismo mental requería de la asimilación de muchos de los métodos y técnicas procedentes de estas concepciones que, no obstante su descrédito por parte de la academia, seguían latiendo en los laberintos explicativos de los victorianos ${ }^{12}$.

\section{Conclusión}

¿Es epistémicamente fértil presentar a la frenología y a la fisiognomía como sistemas de creencias y prácticas que debían perecer en el curso del progreso inexorable de una ciencia más objetiva y más racional? ¿Es historiográficamente prudente estimar a los ingleses victorianos como pueriles y crédulos ante la circulación de un conocimiento tan alevosamente cargado de prejuicios? ¿Pueden jactarse las ciencias sociales (psicología, psiquiatría, criminología, antropología forense, entre otras) de haber abandonado definitivamente el terreno de los esencialismos? Cada uno de estos interrogantes ha de ser tratado con cautela. Conviene, al respecto, tener en cuenta varios aspectos, de los cuales se mencionarán los más importantes.

En primera instancia, hay que decir que el trato despectivo de la fisiognomía y la

Un examen muy rico de la incorporación de supuestos fisiognómicos y frenológicos en las teorías hereditaristas de fines del siglo XIX se encuentra en la tesis doctoral de John Waller: The social and intellectual origins of Sir Francis Galton's (1822-1911). Ideas on heredity and eugenics. London: University College of London. frenología basado en la apreciación retroactiva de ingenuidad ante lo innegablemente falso menoscaba la posibilidad de entender cómo una cultura construye, fundamenta y usa el conocimiento "científico" como instrumento de legitimación de sus prácticas (las cuales, a su vez, retroalimentan a dichas ideas y teorías). Como se ha intentado sugerir en este artículo, los métodos de codificación y clasificación de los seres humanos de acuerdo a los "indicios" fisiognómicos y frenológicos ilustran la tenacidad de los prejuicios no conscientes (la terquedad de lo latente) y la sorprendente maleabilidad de los datos cuantitativos que se suponían "objetivos" bajo los intereses de una idea preconcebida. Como han sugerido S. Gould (1982), G. Stocking (1987) y otros, los condicionantes culturales parecen ser inevitables a la hora de observar y organizar la experiencia perceptual, por más alegadamente imparcial que el científico pueda declararse. La exposición que hace Stocking de la reacción horrorizada de Darwin ante los fueguinos sudamericanos ilustra cómo la noción de un tipo humano particular se imponía sobre la evidencia que se le presentaba y la modelaba de una manera determinada. Pero este caso de Darwin es quizás extremo, porque ejemplifica el fenómeno de la carga teórica de la observación en la percepción de dos culturas muy diferentes entre sí. ¿Necesitamos ir tan lejos? No necesariamente. La reacción darwiniana era, principalmente, una práctica que reproducía la clase de estereotipificación practicada por los ingleses en su misma sociedad londinense. La clasificación de la población británica en tipos sociales y raciales acometida tanto por antropólogos profesionales como amateurs, seguía exactamente el mismo método darwiniano, que no era sino una imitación del procedimiento del historiador natural que se 
servía de los rasgos físicos y conductuales para distinguir una variedad animal o "raza"13. En consecuencia, cabe decir que el análisis de este condicionamiento perceptual desencadenado por ciencias populares y académicas en el siglo XIX puede arrojar luz sobre el modo de examinar el papel jugado por otros métodos de ordenamiento y clasificación que han dominado a lo largo de la historia, y, en especial, los que dominan nuestra propia época (la psicometría puede ser considerada como caso destacado, pues no hay que olvidar que los frenólogos también estaban muy preocupados por "medir" el grado de inteligencia de cada individuo) ${ }^{14}$.

Por otra parte, el abordaje displicente de estos cuerpos de conocimientos craneológicos (ridiculizados, sobajados o incluso ignorados en las historias oficiales de la psicología científica) ${ }^{15}$ pudiera hacer pensar (erróneamente) que tales sesgos han sido erradicados, y que se ha abandonado irreversiblemente el camino de la pseudociencia en vías del correctivo plan de viaje que sigue el conocimiento racional. Pero este estilo de presentación de los "hechos" es nuevamente capcioso, ya que esa clásica desestimación está sustentada en el equivocado principio según el cual "la anterioridad cronológica es una inferioridad lógica" (de acuerdo a la atinada expresión de

13 Sobre esta analogía, ver Pratt (1992). Acerca de la actitud de Darwin ante los fueguinos, consultar Stocking (1987).

14 Es preciso establecer que, dentro del terreno psicológico en el cual fermentaron las investigaciones psicométricas, no todas las posiciones representan formas de esencialismos. En un abordaje que profundice su excavación en dicha área sería menester considerar, por ejemplo, la importantísima contribución de W. Mischel $(1968,1979)$ acerca de la noción de rasgo, así como el amplio abanico de posturas adoptadas en respuesta a él. Desde la psicología cognitiva, no puede dejar de mencionarse las clásicas contribuciones de Beck (1996, 1999).

Historias oficiales de la psiquiatría son, por ejemplo, Zillboorg (1945) y Alexander y Selesnick (1970).
Canguilhem (1952: 43)). Como bien señala este historiador francés, ahora nos causa humor y poco respeto una idea como la defendida por el neurofisiólogo alemán Paul-Jules Möbius, que en 1907 exaltaba, reviviendo a Gall, la "protuberancia de las matemáticas" (a la cual situaba arriba de la órbita izquierda, en el lado externo). Sin embargo, "parecemos menos dispuestos, en estos tiempos, a reírnos de los cromosomas de los "superdotados" o de la herencia genética del cociente intelectual" (Canguilhem 1980: 82). Dicho en otras palabras, la frenología puso hábilmente en la palestra un tema que hoy reviste la más alta relevancia, y es el de la relación entre mente/cerebro (el cual remite a un problema filosófico de aún mayor envergadura: la relación mente/cuerpo, y la candente controversia de si existe (o no) un gap ontológico entre ambos).

Así, tratar de determinar la incidencia que la frenología tuvo sobre el desarrollo de la ciencia adoptando como criterio la dicotomía verdad/ falsedad refleja una miopía epistemológica e historiográfica muy alentada por el vicio de observar el pasado de la ciencia desde la constrictiva óptica positivista (que impele a idealizar las teorías como si fuesen puras e impermeables a la mimetización cultural). Como acertadamente indica R. Young (1968), un efecto epistémico semejante tiene la intención de distraer nuestra atención respecto a la cuestión realmente importante, que es la de preguntarnos e investigar cómo las fuerzas de la sociedad evocan una cierta teoría científica y cómo ésta llega a enquistarse en los debates culturales, políticos eideológicos. Cabe recordar que, apenas 50 años después de que algunos médicos definieran a la frenología como "un sistema fisiológico-psicológico al que sólo le resta completarse" (Lélut 1836: 239), arribaron 
con paso heroico "las investigaciones de Ferrier, Fritsch, Hitzig y Flechsig para inaugurar "la edad de oro de las localizaciones cerebrales", y con ello dibujar la primera carta topográfica del cerebro" (Canguilhem 1980: 82).

Aquel envión que la craneología disparó estuvo lejos de desvanecerse con el paso del tiempo. Por el contrario, desde 1891 esos conocimientos topográficos se convertirían más tarde en técnica de psicocirugía (acción llamada, desde entonces, lobotomía). A propósito de estas repercusiones, Canguilhem nota, con su típica agudeza, "la rapidez con la cual el supuesto conocimiento de las funciones del cerebro se ha invertido en técnicas de intervención, como si la marcha teórica estuviese congénitamente suscitada por un interés práctico" (1980: 83). Como "ciencia de la mente", la craneología motivó el desarrollo del localizacionismo que estructuró gran parte de la neurología cerebral, y como una "frenología aplicada" funcionó a la manera de un instrumento de orientación y de selección tan exitoso como lo pudiera ser hoy cualquier forma de psicoterapia que pretenda echar a andar su vocación pragmática en el terreno del diagnóstico de aptitudes, de la prevención de la delincuencia, de la salud mental e incluso de la psicopatología. Sus implicaciones materialistas, fatalistas y reduccionistas siguen presentes en otras formas de cosmovisiones intelectuales que la denuncian de "ficticia mitología cerebral" pero que carecen, ellas mismas, de una reflexión epistemológica que les permita elucidar sus propios esencialismos rigidizantes.

Aún hoy existe la marcada tendencia a creer que la explicación de la conducta humana depende de la exploración del individuo, y al servicio de tal objetivo trabaja la impresionante tecnología de la neuroimagen (resonancias magnéticas, electroencefalografía, tomografía axial computada, entre otras innovaciones) así como la promocionada industria de los tests neuropsicológicos. Por amplio y vanguardista que parezca este escenario, el foco de interés para entender la conducta humana sigue estando intacto: es el mismo sujeto individual de la fantasmática craneología. Las teorías explicativas dominantes han cambiado su constructo para poder dar cuenta de por qué un sujeto hace lo que hace (y se invoca a su carácter, su personalidad, su inconsciente automático, su inconsciente afectivo, su cerebro, sus neurotransmisores, sus genes o alguna otra entidad dotada de capacidad explicativa). En ese contexto pletórico de vertientes individualistas (cada una impregnada de sus peculiares prácticas indiciarias) proliferan distintas formas de intervención de la conducta, la mayoría de ellas asumiendo con "naturalidad" que la conducta humana es abordable, entendible y modificable con independencia de las relaciones y de las ecologías interaccionales dentro de las que el sujeto vive. La explicación esencialista de la conducta humana pone en jaque la capacidad de elección y cambio, de la misma forma que la explicación individualista inhibe la posibilidad de observar la conducta humana como un resultado de los procesos interaccionales que los sujetos construyen y reconstruyen permanentemente en sus cambiantes relaciones. Un abultado conglomerado de disciplinas sociales tironean nuevamente al ser humano hacia el cerebralismo (piénsese en el caso de las neurociencias o de la sociobiología), alimentando la dura advertencia de Canguilhem cuando sostenía que "a lo largo del siglo XIX, el "Yo pienso" ha sido, en muchos comentarios, rechazado o refutado en provecho de un pensar sin sujeto personal responsable" (1980: 85). 
Dada la hegemónica presencia de dicha obsesión esencializadora (y su "eterno retorno"), parece improbable que se revierta la abrumante epidemia clasificatoria de los individuos en nombre de una ciencia progresiva, racional y objetiva. Será en los escasos espacios de reflexión crítica donde podrá brotar algún entendimiento alternativo de la conducta humana, tal y como sucede, por ejemplo, con las contribuciones de la epistemología sistémico-relacional ${ }^{16}$.
Mientras tanto, será una vez más la filosofía quien asuma el compromiso de deconstruir y desenmascarar los intrincados mecanismos y procesos por los cuales llegan a instaurarse nuevas configuraciones conceptuales, nuevas expectativas "visuales" y nuevos imaginarios acerca de "lo natural" en la conducta humana.

\section{Bibliografía}

Alexander, F. G. y Sheldon T. S. 1970. Historia de la psiquiatría: Una evaluación del pensamiento y de la práctica psiquiátrica desde la época prehistórica a nuestros días. Barcelona: Espaxs.

Aguirre, C. 2007. "Indicios, lecturas indiciarias, estrategia indiciaria y saberes populares. Una hipótesis sobre los límites de la racionalidad burguesa moderna". Summa Humanitatis 1 (1): 9-44.

Bain, A. 1861. On the study of character, including an estimate of phrenology. London: Parker, Son \& Bourn.

Bateson, G. 1976. Pasos para una ecología de la mente. Buenos Aires: Ediciones Lohl.

Books. 1979. Mente y naturaleza. Nueva York: Bantam

Baucom, I. 1999. Out of place: Englishness, empire and the locations of identity. Princeton: Princeton University Press.

Beamish, R. 1865. Psychonomy of the hand: Or, the hand and index of mental development: According to $\mathrm{Mm}$. D’arpentigny and desbarrolles... London: Pitman. Evelyn.

Beaver, P. 1970. The crystal palace. London: Hugh

Beck, A. 1996. "Beyond belief: A theory of modes, personality and psychopathology". Frontiers of cognitive therapy. Salkovskis, P. (Ed.). New York: Guilford Press. $1-25$.

16 Acerca de referentes en la epistemología relacional se pueden consultar las fuentes del paradigma sistémico, tales como: Bateson (1976, 1979), Keeney (1987), Watzlawick, Beavin y Jackson (1965), Christiansen (2012, 2013, 2014).
1999. "Cognitive aspects of personality disorders and their relation to syndromal disorders: A psychoevolutionary approach". Personality and psychopathology. Cloninger, C. R. (Ed.). Washington: American Psychiatric Press. 411-430.

Best, G. F. A. 1971. Mid-Victorian Britain 1851-1875. London: Weidenfeld and Nicolson.

Brabazon, R. 1881. "The health and physique of our city population". Nineteenth Century X: 80-90.

.1970. Victorian people: A re-assessment of persons and themes. Chicago: University of Chicago Press.

Canguilhem, G. 1952. Lo normal y lo patológico. México D.F.: Siglo XXI.

1980. "Le cerveau et la pensée". Prospective et Santé 14: 81-98.

Carré, J. 1989. "The public park". The later Victorian age (Vol. 7): The Cambridge Guide to the Arts in Britain. Ford, B. (Ed.). Cambridge: Cambridge University Press. 76-85.

Carson, J. C. L. 1868. The fundamental principles of phrenology. London: Houlson \& Wright.

Castiglioni, A. 1947. A history of medicine. New York: Knopf.

Christiansen, M. 2009. La arquitectura del destino. Análisis de la psicología del carácter desde una historiografía contextualista. Guanajuato: Universidad de Guanajuato.

2012. "Las relaciones de poder desde una epistemología sistémica". European Scientific Journal 8 (20): 141-161. 
2013. "Violence and maltreatment in relational ecologies: Toward an epistemology of corresponsability". Interpersona 7 (1): 150-163.

2014. "Sobre la violencia y ciertos vicios epistemológicos. El "crimen pasional" como ejercicio crítico-reflexivo". Revista Vanguardia Psicológica 4 (2): 130-146.

Combe, G. 1825. Elements of phrenology. Edinburgo: Anderson.

1827. Essay on the constitution of man and its relations to external objects. Edinburgo: Neill.

1836. A system of phrenology. Edinburgo: Maclachlan, Stewart \& Anderson.

.1840. Moral philosophy: Or the duties of man considered in his individual, social, and domestic capacities. Edinburgo: Stewart \& Co.

Cowling, M. 1989. The artist as anthropologist: The representation of type and character in Victorian art. Cambridge: Cambridge University Press.

Craig, A. R. 1867. The book of the hand. London: Sampson Low, Son \& Marston.

1884. Your luck's in your hand, or the science of modern palmistry. New York: R. Wirthington.

Curtis, L. P. 1971. Apes and angels: The Irishman in Victorian caricature. Washington: Smithsonian Institution Press. Dent.

Darwin, Ch. R. 1859. On the origin of species. London: relation to sex. London: Murray. 1872. The expression of the emotions in man and animals. London: Murray.

Davis, J. B. y Thurman, J. 1856-1865. Crania Britannica. Delineations and descriptions of the skulls of the aboriginal and early inhabitants of the British Islands: With notices of their other remains. London: Taylor and Francis.

Davidson, A. 2001. "The epistemology of distorted evidence: Problems around Carlo Ginzburg's historiography". The emergence of sexuality: Historical epistemology and the formation of concepts. London: Harvard University Press. 142-177.

Dennis, R.1984. English industrial cities of the nineteenth century. A social geography. Cambridge: Cambridge University Press.

Dyos, H. J. 1982. Exploring the urban past: Essays in urban history. Cambridge: Cambridge University Press. y Wolff, M. (Eds.). 1973. The Victorian city Images and realities. Boston: Routledge \& Kegan Paul.

Elias, N. 1987. El proceso de la civilización.
Investigaciones sociogenéticas y psicogenéticas. México D.F.: Fondo de Cultura Económica.

Elliot, B. 1986. Victorian gardens. Oregon: Timber Press.

Elliotson, J. 1835. Human physiology. Orme: Longman, Rees.

Estrada, C. y Oyarzún, M. 2007. "Teorías implícitas y esencialismo psicológico: Herramientas conceptuales para el estudio de las relaciones entre y dentro de los grupos". Psykhe 16 (1): 111-121.

Fuller, P. 1989. "Fine arts". The later Victorian age (Vol. 7): The Cambridge Guide to the Arts in Britain. Ford, B. (Ed.). Cambridge: Cambridge University Press. 163-209.

Galton, F. 1865. "Hereditary talent and character". Macmillan's Magazine 12: 157-166, 318-327.

1869. Hereditary genius. An inquiry into laws and consequences. London: McMillan \& Co.

Gall, F. 1825. Sur les fonctions du cerveau et sur celles de chacune de ses parties. Paris: Baillière.

Ginzburg, C. 1991. "Checking the evidence: The judge and the historian". Critical Inquiry 18 (1): 79-92.

Gedisa. 1999. Mitos, emblemas e indicios. Barcelona:

Gould, S. J. 1982. The mismeasure of man.

Harmondsworth: Penguin.

Haley, B. 1978. The healthy body and Victorian culture.

Massachusetts: Harvard University Press.

Heuman, G. J. 1981. Between black and white: Race, politics and free coloreds in Jamaica. 1792-1865. London:

Greenwood Press.

Hilts, V. 1982. "Being the laws of hereditary descent:

Phrenological views on inheritance and eugenics". Journal of the History of the Behavioral Sciences 18 (1): 62-77.

Houghton, W. 1957. The Victorian frame of mind, 1830-1870. New Haven: Yale University Press.

Hunter, J. 1867. Newcastle Weekly Chronicle. January 19.

Irvine, W. 1955. Apes and angels. Darwin, Huxley and Evolution. New York: Time Magazine.

James, W. 1978. Leisure and society. New York: Longman.

Jackson, J. W. 1863. Ethnology and phrenology as an aid to the historian. London: Trubner \& Co.

Jimeno, M. 2002. Crimen pasional. Con el corazón en tinieblas. Brasilia: Universidad de Brasilia.

Keeney, B. 1987. La estética del cambio. Barcelona: Paidós.

King, A. D. 1995. The bungalow. Oxford: Oxford University Press. Kingsley, Ch. 1859. Miscellanies. London: J. Parker \& 
Son.

Lelut, F. 1836. ¿Qué es la frenología? París: Delahays. Lubbock, J. 1865. Prehistoric times. London: Longman. .1870. The origin of civilization and the primitive condition of man: mental and social condition of savages. London: Longman, Green.

Mac Dougal, H. A. 1982. Racial myth in the English history: Trojans, Teutons, and Anglo-Saxons. Hanover: University Press of New England.

Mac Lennan, J. F. 1865. Primitive marriage. Edinburgh: Adam and Charles Black.

Mayhew, H. 1849. London labor and the London poor. London: Viking Penguin.

1874. London characters and the humorous side of London life. London: Stanley Rivers \& Co.

y Binny, J. 1862. The criminal prisons of London and scenes of prison life. London: Griffin, Bohon \& Co.

Maudsley, G. 1867. The physiology and pathology of man. London: McMillan.

Medin, D. L. 1989. "Concepts and conceptual structure". American Psychological Association 44 (12): 1469-1481.

Mischel, W. 1968. Personality and assessment. New York: John Wiley.

1979. "On the interface of cognition and personality. Beyond de person-situation debate". American Psychologist 34 (9): 740-754.

Mill, J. S. 1859a. "Bain's psychology". Edinburgh Review 110: 287-321.

1859b. On liberty. London: Longman, Green.

Morton, S. G. 1854. Types of humanity, or ethnological researches: Based upon the ancient monuments, paintings, sculptures, and crania of races and upon their natural, geographical, philological and biblical history. London: Trubner \& Co.

Murray, J. 1870. "On the nervous, bilious, lymphatic, and sanguine the temperaments: Their connection with races in England, and their relative longevity". Anthropological Review XXVIII: 14-28.

Napier, Ch. 1867. Miscellanea Anthropologica. London: Groombridge \& Son.

1870. The book of nature and the book of man. London: John Camden Hotten, Picadilly.

Olsen, D. J. 1986. The city as a work of art: London, Paris, Vienna. New Haven: Yale University Press.

Paget, J. 1856. "Physiognomy of the human form". Quarterly Review 99: 452-490.

Patterson, R. 1854. "Notice of the life and scientific labors of the late Samuel Geo. Morton, M. D". Types of mankind: Ethnological Researches. Nott, C. J. y Gliddon,
G. (Eds.). Philadelphia: Lippincott, Grambo \& Co. xvii-Ivii. Perazzi, P. 2009. "Cartografías corporales: Las pesquisas antropológicas del Doctor Roberto LehmannNitsche. Buenos Aires, 1897-1908". Cuadernos de Antropología Social 29: 121-134.

Pratt, M. L. 1992. Imperial eyes: Travel writing and transculturation. New York: Routledge.

Prichard, J. C. 1843. The natural history of man: Comprising inquiries into the modifying influence of physical and moral agencies on the different of the human family. London: Bailliere.

Rasmussen, S. E. 1934. London: The unique city. Cambridge: M.I.T. Press.

Reader, W. J. 1964. Life in Victorian England. London:

B.T. Batsford.

Richardson, B. W. 1876. Diseases of modern life. New

York: Appleton \& Company.

Seeley, J. R. 1971. The expansion of England. Chicago: Chicago University Press.

Singer, Ch. J. y Underwood, E. A. 1962. A short history of medicine. Oxford: Clarendon Press.

Spencer, H. 1855. The principles of psychology. London: Longman.

Spurzheim, J. G. 1821. A view of the elementary principles of education. London: Kessinger Publishing. 1825. A view of the philosophical principles of phrenology. London: Knight

Stedman-Jones, G. 1971. Outcast London. A study in the relationship between classes in Victorian society. London: Penguin.

Stocker, R. D. 1900. Physiognomy ancient and modern. London: Simpkin, Marshall, Hamilton, Kent \& Co.

Stocking, G. 1987. Victorian anthropology. London: Collier Macmillan.

Thompson, F. M. L. 1988. The rise of respectable Society: A social history of Victorian Britain, 1830-1900. Massachusetts: Harvard University Press.

Tytler, G. 1982. Physiognomy in the European novel. Princeton: Princeton University Press.

Urteaga, L. 1993. "La teoría de los climas y los orígenes del ambientalismo". Cuadernos Críticos de Geografía Humana XVIII (99). En línea, disponible en: http://www. ub.edu/geocrit/geo99.htm

Van Wyhe, J. 2002. "The authority of human nature: The Schädellehre of F. J. Gall". British Journal for the History of Science 35: 17-42.

2003. "George Combe's of man and the law of hereditary descent". A cultural history of heredity II: 18th and 19th centuries. Rheinberger, H. J. y Müller-Wille, S. (Eds.). Berlin: Max Planck Institut für Wissenschaftsgeschichte. 
165-174.

Waller, J. 2001. The social and intellectual origins of Sir Francis Galton's (1822-1911). Ideas on heredity and eugenics. London: University College of London.

Watkin, D. 1982. The English vision. New York: Harper \& Row.

Warwick, E. 1864. Notes in noses. London: Richard Bentley.

Watzlawick, P., Beavin, J. H. y Jackson, D. D. 1965. Teoría de la comunicación humana. Interacciones, patologías y paradojas. Barcelona: Editorial Herder.
Wells, S. R. 1866. New system of physiognomy. New York: Fowler \& Wells.

Woodward, S. L. 1962. The age of reform 1815-1870. Oxford: Oxford University Press.

Youmans, E. L. 1867. The culture demanded by modern life: A series of addresses and arguments on the claims of scientific education. New York: Wheeler.

Young, R. M. 1968. "Freud: Scientist and/or humanist". Human Nature 7: 7-35.

Zilboorg, G. 1945. Historia de la psicología médica. Buenos Aires: Hachette. 
